\title{
Extracciones dentarias en pacientes en tratamiento con anticoagulantes: revisión de la literatura ${ }^{1}$
}

\author{
Visintini $E^{*}$, Contardo L*, Biasotto $M^{* *}$, Vignoletti $F^{* * *}$
}

\section{RESUMEN}

Objetivo del trabajo. En pacientes en terapia con anticoagulantes orales, una simple extracción puede provocar hemorragias importantes. En la literatura se describen dos enfoques terapéuticos en casos de intervenciones quirúrgicas en esta categoría de pacientes. Uno propone la reducción de la dosis terapéutica del fármaco, mientras que el segundo sugiere la sustitución con heparina. El objetivo de este trabajo es revisar la literatura que trata este tema con el fin de obtener un protocolo ideal.

Conclusiones. En caso de extracciones simples es suficiente usar anestesia con vasoconstrictor (en el caso de que no haya otras contraindicaciones), realizar suturas bien tensadas, aplicar coadyuvantes hemostáticos locales y ácido tranexámico tópico. Solo en casos de intervenciones más complejas, o con valores de INR mayores a 3,5, será necesario reducir la dosis del fármaco y consecuente sustitución con heparina de acuerdo a las instrucciones del hematólogo y del cardiólogo.

Palabras clave: Anticoagulantes, extracciones dentarias, cicatrización.

\section{SUMMARY}

Aim of the work. In patients treated with oral anticoagulants, even simple tooth extractions can produce large hemorrhages. There are two main therapeutic outlines suggested in literature in case of little oral surgery. The first one is based on the reduction of the therapeutic dose of the drug, and the second one on the substitution with heparin. The aim of this paper was to review the.literature on the management of patients treated with anticoagulants in order to obtain univocal guidelines.

Conclusion. In case of simple tooth extractions generally a vasoconstrictive anaesthetic (if there are no other contraindications), tight sutures, the application of hemostats and tronexamic acid are enough. Only in case of more complex surgery or of INR values higher than 3,5 it is necessory to reduce the anticoagulant dose or to change the therapy with heparin, if agreed by the hoemotologist and/ or the cardiologist.

Key words: Anticoagulant drugs, extraction, healin, dental.

Aceptado para publicación: Febrero 2006.

$1 \quad$ Publicado en Doctor OS. 2006; Feb. 17(2): 125.

* $\quad$ Università degli Studi di Trieste. U.C.O. di Clinica Odontoiatrica e Stomatologia.

** Investigador Università degli Studi di Trieste. U.C.O. di Clinica Odontoiatrica e Stomatologia.

*** Master de Periodoncia. Universidad Complutense de Madrid.

Visitine E, Contardo L, Biasotto M, Vignoletti F. Extracciones dentarias en pacientes en tratamiento con anticoagulantes: revisión de la literatura. Av. Odontoestomatol 2006; 22 (4): 241-245. 


\section{INTRODUCCIÓN}

Es cada vez más frecuente el tratamiento de pacientes en tratamiento con fármacos anticoagulantes. Estos fármacos se utilizan en concreto en la profilaxis de las trombosis venosas profundas, del ictus y de las embolias en pacientes que padecen fibrilación atrial, patologías de las válvulas cardiacas o portadores de prótesis valvulares $(1,2)$. Visto el riesgo significativo de episodios hemorrágicos incluso graves, son necesarios controles periódicos para averiguar que los valores de las pruebas analíticas estén dentro del rango terapéutico del fármaco.

Simples extracciones pueden determinar graves hemorragias no controlables, incluso a la hora de la infiltración con anestesia, con consecuentes tumefacciones que pueden provocar disfagia y tal vez dificultades respiratorias (3). En literatura se han propuesto dos protocolos terapéuticos: suspensión del fármaco (4-6) reducción de la dosis o sustitución del principio activo con moléculas más fáciles de antagonizar en casos de hemorragias profusas (5-8).

Hoy en día la mayoría de los autores concuerdan que la suspensión de la terapia anticoagulante es peligrosa, causando un aumento inmediato de la capacidad de coagulación y consecuente riesgo de fenómenos tromboembólicos (9). Por tanto es necesario de acuerdo con el cardiólogo, reducir la dosis del fármaco manteniendo valores mínimos de rango terapéutico (10).

Objetivo de este trabajo es describir un protocolo basado en una revisión de la literatura sobre el tratamiento de pacientes en terapia anticoagulante.

\section{ANTICOAGULANTES ORALES}

Los anticoagulantes orales son antagonistas de la vitamina K. Los factores II, VII, IX, X de la cascada de la coagulación y las proteínas $\mathrm{C}$ y S, son biológicamente inactivos a menos que residuos de ácido glutámico no sean carboxilados por un sistema enzimático microsomal que utiliza como factor la vitamina $\mathrm{K}$ reducida.
El suministro de una cantidad suficiente de vitamina $\mathrm{K}$ es capaz de antagonizar dosis incluso elevadas de anticoagulantes orales (11).

Dosis terapéuticas de warfarin reducen de un 30$50 \%$ la cantidad total disponible de cualquier factor vitamina $\mathrm{K}$ dependiente producidos por el hígado; además las moléculas secretadas están hipercarboxiladas, con una consecuente disminución de la actividad biológica (10-40 \% menos de un sujeto que no está en terapia).

La biodisponibilidad de la solución de warfarin sódico racémico es casi completa cuando se suministra el fármaco por vía oral, intramuscular, endovenosa o rectal. Se encuentra en el plasma una hora tras la toma y las concentraciones alcanzan el valor máximo en 2-8 horas. El warfarin se liga casi por completo a las proteínas plasmáticas (99\%), principalmente la albúmina, y se distribuye rápidamente en un volumen igual al espacio ocupado por la albúmina. Se trasforma posteriormente en metabolitos inactivos que se excretan con la orina o las heces. La hemivida del fármaco varía entre 20 y 60 horas, con un valor medio de 40 .

El warfarin sódico se presenta en comprimidos de: 2; 2,5; 5; 7,5 y $10 \mathrm{mg}$ de principio activo. En los adultos la dosis es de 10-15 mg por día durante 2-4 días, seguidos por 2-15 mg/día dependiendo de los valores de INR. El fármaco se debe tomar a la misma hora cada día; el momento de acostarse parece ser el más apropiado por el efecto máximo que se ha observado en las muestras del día siguiente. No están indicadas dosis elevadas de ataque pues reducen el tiempo necesario para obtener su efecto y aumentan la toxicidad (10).

Se deben considerar potencialmente peligrosas sustancias o condiciones que alteran la captación o el metabolismo del anticoagulante oral o de la vitamina $\mathrm{K}$, o las que alteran la síntesis, función o eliminación de los factores involucrados en el proceso de fibrinólisis o hemostasis así como las condiciones que influyen en la integridad de la superficie epitelial. Una alteración del PT en caso de toma de anticoagulantes puede deberse a: reducción de la adsorción del fármaco causada por la unión con colestiramina en el sistema digestivo; hipoproteinemia por síndrome nefrótico; aumento de la eliminación metabólica del fár- 
maco secundaria a una activación de enzimas hepáticos por barbitúricos, rifampicina, fenitoína o ingestión crónica de alcohol; ingestión de grandes cantidades de comida o de integradores vitamínicos; aumento de la concentración de factores de la coagulación por embarazo. En estos casos el PT será más corto (11).

Una reducción de los factores de la coagulación se puede deber a alteración de la función hepática, insuficiencia cardíaca o estados hipermetabólicos. Generalmente estos factores contribuyen a la prolongación del PT. Interacciones graves que no alteran el PT se deben a la inhibición de la función plaquetaria, por la acción de fármacos como el ácido acetilsalicílico, por gastritis y el ulcera inducidos por fármacos antiinflamatorios (11).

La hemorragia es el principal efecto adverso de los anticoagulantes orales. Los episodios particularmente graves ocurren en áreas en las que se pueden producir daños irreversibles por efecto de compresión de estructuras vitales (como por ejemplo hemorragia intracraneal o pericárdica) o de una hemorragia interna masiva que no se puede diagnosticar inmediatamente. El riesgo de un hematoma cerebral o subdural puede aumentar hasta 10 veces en un paciente de cincuenta años en tratamiento con anticoagulantes. Cada procedimiento que puede provocar sangrado debe considerarse atentamente y hay que estar preparados a tratar eventuales episodios hemorrágicos. Si un paciente presenta cualquier signo de hemorragia, se debe suspender el suministro de la dosis sucesiva de anticoagulante y averiguar el INR (10).

En presencia de una hemorragia continua, la vitamina $\mathrm{K}_{1}$ es un antídoto eficaz.

Si fuera necesaria una competencia hemostática inmediata se pueden reestablecer las concentraciones adecuadas de factores de la coagulación vitamina-K dependientes a través de una transfusión de plasma congelado. Existen además concentrados de proteínas K-dependientes.

\section{DICUMAROLES}

Son anticoagulantes indirectos que presentan una estructura parecida a la vitamina $\mathrm{K}$ y compiten con ella inhibiendo la síntesis hepática de factores K-dependientes como la protrombina y los factores VII, IX y X. Estos fármacos presentan un periodo de latencia bastante largo de aproximadamente 18 horas. Los más utilizados son el warfarin sódico (Coumadin) y el acecumarol (Sintrom). El antídoto natural es la vitamina K (Konakion) (11). Hay que tener en cuenta que diferentes fármacos interaccionan con los dicumaroles aumentando o reduciendo su actividad (tabla 1).

\section{MANEJO DEL PACIENTE EN TERAPIA ANTICOAGULANTE}

Existen dos protocolos descritos en literatura que son la reducción/sustitución o la suspensión del anticoagulante. Suspender la terapia en pacientes de alto riesgo puede provocar tromboembolias (6). Sustituyendo el warfarin con la heparina, es siempre necesario ingresar el paciente para monitorizarle durante el postoperatorio en caso de que se provoquen hemorragias. Bloomer (8).

Hoy en día se tiende cada vez mas a mantener la terapia normal y a controlar el riesgo de hemorragias con coadyuvantes hemostáticos tópicos $(5,6,8)$.

Se debe evaluar el tipo de intervención: se tratará de bajar el INR en caso de tratamientos complejos (5) y,

\section{TABLA 1}

Reducción actividad dicumaroles

Antibióticos (tetraciclinas, cotrimoxazol, eritromicina, metronidazol, cipro-floxaciclina, isoniazide), antimicóticos (fluconazol, itraconazol), chinidina, alcohol, metiltiouracile, isótopos radioactivos, fenilbutazona, aspirina, indometacina, contraceptivos, simvastatina, tamoxifeno, piroxicam, propafenone, propanolol, omeprazol, amiodarone e vacuna antigripal.

\section{Reducción actividad dicumaroles}

Barbitúricos, meprobamato, griseofulvina, contraceptivos, carbamacepina, clor-diazepossido y rifampicina. 
en caso de un paciente de riesgo alto, se suspenderá la terapia normal sustituyéndola con heparina y hospitalizando el paciente (8). En el caso de extracciones simples de 1-3 elementos dentarios, si el valor de INR es superior a 4 es posible hacer la intervención con las siguientes precauciones. Se infiltra anestesia con vasoconstrictor (excepto contraindicaciones de tipo médico) y a continuación se hace la extracción con fórceps o botador, tratando de causar el mínimo trauma a los tejidos. Se debe hacer un desbridamiento de los alveolos en casos de periodontitis severa, ya que en estos casos es cuando se han descrito las mayores hemorragias. Blinder (12).

Finalmente se pasa a la fase de sutura, utilizando preferiblemente material reabsorbible que, al acumular menos placa, reduce el riesgo de inflamación de los tejidos y de la posterior hemorragia (5); se utilizan además tapones hemostáticos tópicos como celulosa oxidada regenerada, esponjas de gelatina reabsorbible, colágeno o fibrina (13). Algunos autores sugieren el uso de PRP como coadyuvante de la coagulación (14). Se considera útil el uso de gasas con ácido tranexámico.

Durante el postoperatorio el paciente deberá abstenerse de comida caliente y tabaco, aplicando hielo y en caso de sangrado debe aplicar presión con una gasa con ácido tranexámico; de acuerdo a Carter, se aconsejan enjuagues con el mismo fármaco evitando la ingestión (12). El paciente tiene que tener la posibilidad de contactar un especialista en caso de una hemorragia profusa. En caso de que haya que suspender la terapia por tratamientos más complejos, se debe sustituir con heparina subcutánea en dosis bajas (5.000 UI) cada 12 horas y programar la intervención al tercer día. En este periodo se debe controlar el número de plaquetas, teniendo en cuenta que el 1,3\% de los casos puede manifestar citopenia, especialmente en pacientes con historia pasadas de problemas similares (8). Tras la intervención se deberá volver a las dosis normales de terapia anticoagulante.

\section{FÁRMACOS ANTIAGREGANTES}

Las plaquetas provocan un tapón hemostático inicial en las lesiones vasculares; además participan en las reacciones que desencadenan arteriosclerosis y trombosis. Para antagonizar estas funciones patológicas de las plaquetas se utilizan fármacos específicos:

- Ácido acetilsalicílico. El tromboxano A2 es un inductor importante de la agregación plaquetaria y un potente vasoconstrictor. El ácido acetilsalicílico bloquea la producción de tromboxano A2 mediante unión covalente con la cicloxigenasa; debido a que las plaquetas son estructuras anucleadas, no pueden volver a producir la enzima y permanecen inactivas durante el resto de su vida media (entre siete y diez días). (11).

- Ticlopidina. Inhibe la función plaquetaria induciendo un estado parecido a la trombo astenia. Interfiere con la unión del fibrinógeno inducida por el ADP a la membrana de la plaqueta en determinados lugares receptores. Como consecuencia, se inhiben la adhesión y la agregación plaquetaria. Alarga el tiempo de sangrado y el efecto máximo se observa después de varios días de tratamiento. La disfunción plaquetaria persiste durante varios días tras la suspensión del fármaco (11).

\section{MANEJO DEL PACIENTE CON ANTIAGREGANTES PLAQUETARIOS}

Normalmente no se debe modificar la pauta terapéutica. Los pacientes en tratamiento con dosis de ácido acetilsalicílico superiores a los $100 \mathrm{mg}$ al día pueden presentar sangrados anormales, así que se sugiere el uso de tapones hemostáticos tópicos y una sutura muy cerrada (5).

\section{BIBLIOGRAFIA}

1. Akhtar W. Reeves WC, Movahed A. Indications for anticoagulation in atrial fibrillation. Am Fam Physician. 1998 Jul;58(1):130-6.

2. Kuntze CE, Blackstone EH, Ebels T. Thromboembolism and mechanical heart valves: a randomized study revisited. Ann Thorac Surg. 1998 Jul;66(1):101-7 
3. Horlocker TI. Wedel DJ, Benzon H, Brown DL, Enneking FK, Heit JA, Mulroy MF, Rosenquist RW, Rowlingson J, Tryba M, Yuan CS. Regional anesthesia in the anticoagulated patient: defining the risk. the second ASRA Consensus Conference on Neuraxial Anesthesia and Anticoagulation L Reg Anesth Pain Med. 2003 May-Jun;28(3):172-97.

4. Souto JC, Oliver A, Zuazu-Jausoro L Vives A, Fontcuberta J. Oral surgery in anticoagulated patients without reducing the dose of oral anticoagulant: a prospective randomized study. J Oral Maxillofac Surg. 1996 Jan;54(1):27-32; discussion 323.

5. Scully C, Wolff A. Oral surgery in patients on anticoagulant therapy. Oral Surg Oral Med Oral Pathol Oral Radiol Endod. 2002 Jul;94(1):57-64.

6. Devani P, Lavery KM, Howell CJ. Dental extractions in patients on warfarin: is alteration of anticoagulant regime necessary? $\mathrm{Br} \mathrm{J}$ Oral Maxillofac Surg. 1998 Apr;36(2):107-11.

7. Roser SM, Rosenbloom S. Continued anticoagulation in oral surgery procedures. Oral Surg Oral Med Oral Pathol. 1975 Oct;40(4):448-57.

8. Sloomer CR. Excessive hemorrhage after dental extractions using lowmolecular-weight heparin (Lovenox) anticoagulation therapy. J Oral Maxillofac Surg. 2004 Jan;62(1):101-3.

9. Todd DW, Roman A. Outpatient use oF low-molecular weight heparin in an anticoagulated patient requiring oral surgery: case report. J Oral Maxillofac Surg 2001 Sep;59(9):1090-2; discussion. 1092-3.

10. Jeske AH, Suchko GD, ADA Council on Scientific Affairs and Division of Sciences. Journal ofthe American Dental Research. Lack of a scientific basis for routine discontinuation of oral anticoagulation therapy before dental treatment. J Am Dent Assoc. 2004;135(1):28.

11. Goodman $\mathcal{E}$ Gilman. Le basi farmacologiche della terapia. Ed it. a cura di Sirtori-Govoni. Milano: McGraw- Hill. 1997: p. 223-41.

12. Blinder D, Manor Y, Martinowitz U, Taicher S. Dental extractions in patients maintained on oral anticoagulant therapy: comparison of INR value with occurrence of postoperative bleeding. Int J Oral Maxillofac Surg. 2001 Dec;30(6): 518-21.

13. Carter G, Alastair G, Lloyd J. Tranexamic acid mouthwash versus autologous fibrin glue in patients taking warfarin undergoing dental extractions: a randomized prospective clínical study. J Oral Maxillofac Surg. 2003;61:1432-5.

14. Della Valle A, Sammartino G, Marenzi G, Tia M, Espedito di Lauro A, Ferrari F, Lo Muzio L. prevention of postoperative bleeding in anticoagulated patients undergoing oral surgery: use of plateletrich plasma gel. J Oral Maxillofac Surg. 2003 Nov;61(11):1275-8. 